\title{
ADAPTIVE COMMUNICATION SYSTEM DESIGN FOR HF/VHF MULTIPLE-MECHANISM PROPAGATION PATHS
}

\author{
M. Darnell* \\ B. Honary ${ }^{\dagger}$ \\ G. Vongas \\ University of Hull, UK. \\ University of Warwick, UK. \\ DRA Farnborough, UK.
}

\begin{abstract}
The paper describes the nature of multiple-mechanism propagation paths (MMPPs) in the HF/low-VHF frequency range from $2-200 \mathrm{MHz}$. The design of systems to exploit such paths is then considered: channel encoding/decoding and real-time channel evaluation (RTCE) aspects are emphasised. Finally, the overall architectural framework for an MMPP radio system, currently under development, is presented.
\end{abstract}

\section{Multiple Mechanism Paths \& Digital Signal Processing}

Modern radio communication systems are normally designed to exploit a given propagation mechanism in order to meet their operational requirements. This means that the parameters of such systems are, to a large extent, dictated by the characteristics of a given propagation mechanism. Currently, most practical radio systems make use of fixed signal formats and control procedures. If a system is adaptive in response to channel conditions, as sensed say by real-time channel evaluation (RTCE), then the nature of that adaptation will be determined by the range of variation expected of the specified mechanism.

The design philosophy outlined above is somewhat artificial in that there are many types of radio path which do not exhibit a single dominant propagation mechanism; rather, a number of distinct mechanisms with very different characteristics may exist - possibly simultaneously. Consequently, these additional mechanisms will tend to be regarded effectively as noise/interference by a communication system designed for a specific mechanism. Examples of this include microwave line-of-sight (LOS) paths over which tropospheric propagation can also occur, HF surface wave paths with skywave interference, meteor-burst paths with background

"Department of Electronic Engineering, University of Hull, Hull, HU6 7RX, UK. (Email: miked@comms.ee.hull.ac.uk)

tDepartment of Engineering, University of Warwick, Coventry, CV4 $7 \mathrm{AL}, \mathrm{UK}$

tDRA Aerospace Division, RAE, Farnborough, GU14 6TD ionospheric scatter, etc. In the part of the radio spectrum up to say $200 \mathrm{MHz}$, the situation may be extremely complex, as will be demonstrated in this paper. Nevertheless, from an operational viewpoint this HF/low-VHF region is still of great importance; consequently, every available method should be sought to improve the availability and reliability of LOS and beyond-LOS (BLOS) communication systems using this range of radio frequencies. An obvious starting point is to take a pragmatic view of the nature of the radio path to be employed; Section 2 below attempts to do this.

In parallel with this consideration of the radio path, an assessment needs to be made of the technology now available to implement radio systems. Over recent years, there have been major changes in the signal generation and processing techniques and devices available to the communication system designer; as yet, however, these changes have not affected the basic nature of operational systems. The reasons for this are simple to identify:

(i) the time taken for new designs to become operational is relatively long - typically a minimum of several years;

(ii) there is a tendency for designers to still think in "conventional" terms, ie implementing functions in an essentially analogue manner;

(iii) the fundamental nature and potential of the propagation medium, particularly at the lower frequencies below about $100 \mathrm{MHz}$, is not yet fully appreciated.

There is a need for design thinking to become digitally oriented; signal generation and processing algorithms and techniques implemented in digital form must exploit the unique capabilities of digital signal processing (DSP) devices, rather than simply replicating functions previously implemented in analogue form.

\subsection{1}




\section{The Nature Of Propagation In The Band Up To $200 \mathrm{MHz}$}

The individual propagation mechanisms that can occur in the band up to $200 \mathrm{MHz}$ are now reviewed briefly by means of the Table 1. Operationally, their LPI/AJ properties are important; these are quantified approximately in Table 1 for each individual mechanism.

In this list of propagation mechanisms, the phenomenon of "ducting" arises from atmospheric refractive index variations close to the earth's surface which can trap radio waves in ducts, analogous to waveguides. Since waveguide propagation tends to be low-loss, this form of energy can allow radio waves to propagate at relatively high levels over distances considerably in excess of LOS. Ducting is comparatively unpredictable, its incidence being correlated to some extent with climatic conditions. References 1 to 7 describe the characteristics of these propagation mechanisms in more detail.

All the entries in Table 1 correspond to well known and reasonably well characterised mechanisms in the band up to $200 \mathrm{MHz}$. The only exception to this is tropospheric scatter which is normally associated with high-capacity transmission systems at considerably higher frequencies. However, recent work [8] [9] has shown that troposcatter is a viable means of information transfer at much lower frequencies, down to $30 \mathrm{MHz}$ and below.

\section{Channel Encoding and RTCE For Multiple-Mechanism Propa- gation Paths}

The range of the mechanisms in an MMPP will give rise to received signals with a wide variability in their characteristics. This variability will manifest itself to the system user as a channel capacity which changes with time. If a radio system is to make effective use of this variable capacity, its parameters must be adapted in response to the channel state at any time. This section now examines channel encoding/decoding techniques that are designed for such an environment. To initiate the necessary adaptation, the channel state must be quantified by means of appropriate RTCE procedures, preferably integrated with the channel encoding procedures.

\subsection{Channel Encoding}

In the context of the type of radio system being discussed, channel encoding will be taken to encompass the following functions: (a) synchronisation; (b) error control; (c) modulation; (d) multi-user coding; (e) security.
A unifying concept which coalesces the above functions is termed "multi-functional coding" [10]. Here, it is assumed that the receiver will possess a certain level of processing power. In an adaptive system, the major practical problem is how best to assign this power between the various channel coding functions in order to optimise the overall system performance with respect to the user requirements.

\subsection{Synchronisation}

Arguably, the most important channel encoding function in a digital transmission system is that of synchronisation; without effective synchronisation, the system cannot operate at all. In an MMPP, one important effect that can occur is a rapid change in propagation delay, due to a change in dominant mechanism. If this change does not exceed one symbol interval, then conventional synchronisation tracking arrangements such as delay-lock loops can cope successfully. Should the delay change be greater than one symbol interval, however, the system may need to reacquire symbol synchronisation. For MMPPs, therefore, it would appear necessary to incorporate an on-going "fly-wheel" synchronisation mode, together with an integral acquisition mode, embedded at intervals within the transmitted data. Also, it is important that both symbol and block synchronisation are maintained.

In reference [11], two techniques, known as modulationderived synchronisation (MDS) and code-derived synchronisation (CDS), are described; these allow the extraction of synchronisation information from the normal operating signals of a radio system by means of specific DSP algorithms. In the case of MDS, the output of a quadrature digital matched filter is monitored at each sampling instant; the output of this matched filter then provides a robust timing waveform at the symbol rate. With CDS, the received signal is applied with all possible codeword time shifts to the error control decoder where it is compared with all possible codewords; at the condition corresponding to the correct codeword and shift, the decoder output will be a minimum - assuming that the error control power of the code has not been exceeded; this is information that can be used for block synchronisation purposes.

\subsection{Embedded Error Control and Modula- tion}

In a variable channel capacity environment, it is important that both error control and modulation procedures can adapt to the channel state. Embedded coding [12] and embedded modulation [13] have the potential to do this. In both cases, the signal format simultaneously contains elements that have different levels of resilience to noise and distortion. When the received SNR is high, all elements can be recovered successfully; as the SNR degrades, only the 


\begin{tabular}{|c|c|c|c|c|}
\hline Mechanism & Range & Variability & Availability & $\begin{array}{l}\text { LPI/AJ } \\
\text { Properties }\end{array}$ \\
\hline $\begin{array}{l}\text { Surface wave } \\
\text { (more efficient } \\
\text { at lower } \\
\text { frequencies }\end{array}$ & $\begin{array}{l}500 \mathrm{~km} \\
\text { (over sea) } \\
\text { (depends on } \\
\text { ground } \\
\text { constants) }\end{array}$ & $\begin{array}{l}\text { amplitude: } \\
\text { small/phase: } \\
\text { some }\end{array}$ & high & good \\
\hline $\begin{array}{l}\text { Normal } \\
\text { skywave (via } \\
\text { ionospheric } \\
\text { refraction) }\end{array}$ & $\begin{array}{l}\text { out to world- } \\
\text { wide (depends } \\
\text { on iono- } \\
\text { spheric } \\
\text { properties) }\end{array}$ & $\begin{array}{l}\text { amplitude: } \\
\text { high/phase: } \\
\text { high/ } \\
\text { frequency: } \\
\text { small }\end{array}$ & $\begin{array}{l}\text { variable } \\
\text { (depends on } \\
\text { system and } \\
\text { path } \\
\text { parameters) }\end{array}$ & poor \\
\hline $\begin{array}{l}\text { Sporadic-E } \\
\text { layer } \\
\text { (sporadic } \\
\text { ionisation } \\
\text { at E-layer } \\
\text { height) }\end{array}$ & $\begin{array}{l}\text { out to about } \\
2000 \mathrm{~km}\end{array}$ & $\begin{array}{l}\text { amplitude: } \\
\text { high/phase: } \\
\text { high/ } \\
\text { frequency: } \\
\text { small }\end{array}$ & $\begin{array}{l}\text { variable } \\
\text { (depends on } \\
\text { location, } \\
\text { season, etc) }\end{array}$ & fair \\
\hline $\begin{array}{l}\text { Meteor-burst } \\
\text { (ionised } \\
\text { meteor } \\
\text { trails at } \\
\text { about } 100 \mathrm{~km}\end{array}$ & $\begin{array}{l}\text { from about } \\
250 \text { to } \\
2000 \mathrm{~km}\end{array}$ & $\begin{array}{l}\text { amplitude: } \\
\text { high/phase: } \\
\text { variable } \\
\text { frequency: } \\
\text { variable } \\
\text { (fading in } \\
\text { tail) }\end{array}$ & $\begin{array}{l}\text { high - but } \\
\text { intermittent } \\
\text { ( } 1 \% \text { duty } \\
\text { cycle) }\end{array}$ & $\begin{array}{l}\text { very } \\
\text { good }\end{array}$ \\
\hline $\begin{array}{l}\text { Ionospheric } \\
\text { scatter } \\
\text { (ionospheric } \\
\text { irregularities } \\
\text { at about } 100 \mathrm{~km} \text { ) }\end{array}$ & $\begin{array}{l}\text { from about } \\
250 \text { to } \\
2000 \mathrm{~km}\end{array}$ & $\begin{array}{l}\text { amplitude: } \\
\text { high/phase: } \\
\text { high/ } \\
\text { frequency: } \\
\text { small (low } \\
\text { level due to } \\
\text { scatter loss) }\end{array}$ & $\begin{array}{l}\text { high - if } \\
\text { ERP } \dagger \\
\text { sufficient }\end{array}$ & poor \\
\hline $\begin{array}{l}\text { Tropospheric } \\
\text { scatter } \\
\text { (tropospheric } \\
\text { irregularities } \\
\text { at a few km) }\end{array}$ & $\begin{array}{l}\text { out to about } \\
400 \mathrm{~km}\end{array}$ & $\begin{array}{l}\text { amplitude: } \\
\text { high/phase: } \\
\text { high/ } \\
\text { frequency: } \\
\text { small (low } \\
\text { level due to } \\
\text { scatter loss) }\end{array}$ & $\begin{array}{l}\text { high - if } \\
\text { ERP } \dagger \\
\text { sufficient }\end{array}$ & fair \\
\hline Line-of-sight & $\begin{array}{l}\text { few } 10 \text { s of } \\
\text { km maximum } \\
\text { (depends on } \\
\text { antenna } \\
\text { height }\end{array}$ & $\begin{array}{l}\text { amplitude: } \\
\text { small/ } \\
\text { frequency: } \\
\text { small/ } \\
\text { phase: small }\end{array}$ & $\begin{array}{l}\text { very } \\
\text { high }\end{array}$ & good \\
\hline Ducting & $\begin{array}{l}\text { out to a } \\
\text { few } 100 \mathrm{~s} \\
\text { of } \mathrm{km}\end{array}$ & $\begin{array}{l}\text { amplitude } \\
\text { variable/ } \\
\text { frequency: } \\
\text { small/phase } \\
\text { variable }\end{array}$ & $\begin{array}{l}\text { unpredictable: } \\
\text { depends on } \\
\text { location and } \\
\text { met conditions }\end{array}$ & good \\
\hline
\end{tabular}

Table 1: Propagation Mechanism Descriptions 45.3.3 
more robust elements can be received successfully. In this way, the effective information transmission rate is matched to the prevailing channel state. Typically, an ARQ protocol would be used in conjunction with these embedded schemes. To date, embedded schemes have been designed primarily with a multiple frequency-shift-keyed (MFSK) modulation format in mind. Such a modulation format is fundamentally robust in the $\mathrm{HF}$ environment, as demonstrated by the PICCOLO system. It also has the advantage that it can be made adaptive by varying the number of tones and symbol duration in response to channel conditions.

\subsection{Real-Time Channel Evaluation}

In order to control the parameters of adaptive channel encoding schemes of the type described previously, it is necessary for data on current channel state, ie RTCE information, to be available in a convenient form. The trend in RTCE systems is now to attempt to extract as much information as possible on channel state from the normal operating signals and standard elements of the radio communication system, eg from demodulators, decoders, synchronisers, protocol activity, etc; these techniques are collectively termed "embedded" RTCE procedures [14]. A further advantage of embedded RTCE is that the information required can be extracted simply and naturally from systems implemented using DSP devices.

\section{Implications Of DSP-Based Ar- chitectures}

\subsection{General Nature of the System Archi- tecture}

The digital processing power available within the architecture of the radio system terminals will be substantial. It is important that this processing power is employed in the most effective manner possible. The processing power is typically of two types:

(i) a PC-based component, normally appropriate for control functions;

(ii) a real-time DSP component, normally used for signal generation and processing (this will comprise multiple DSP devices).

Functions which can be performed by the PC component include:

(a) protocol generation;

(b) signal routing; (c) TX/RX mode and parameter control;

(d) running embedded propagation analysis models $[18]$

(e) control of channel encoding/decoding functions in a multi-functional framework;

(f) RTCE and trend analysis.

The real-time DSP component would tend to be dedicated to (e) above, and would have its power distributed optimally between the functions of synchronisation, error control coding/decoding, modulation/demodulation, multi-user coding/decoding, etc in response to channel state and operational requirements - as indicated in the previous section. The DSP component can also be used for active and passive RTCE when not in use for other purposes, eg as a passive monitor.

\subsection{System Parameter Optimisation}

There is thus an overall optimisation problem to be solved in a systematic manner. How should the available processing power be employed to best meet the user's requirements at any time? For example, what proportion of time and processing power should be spent in actually generating and processing signals, and what proportion spent in say passive monitoring and RTCE so that the transmission and reception process can be more efficient when it does occur? Similarly, more sophisticated and adaptive transmission protocols, whilst consuming more processing power, may actually reduce the transmission time required and hence improve the security of the system. These are problems which are more difficult to solve, and yet have greater significance, with highly variable MMPPs. ERP, achieved via a combination of transmitter power and antenna gain, can also be the subject of adaptive control to enhance LPI/AJ properties.

\subsection{Frequency Management Strategy}

The problems of frequency management in an MMPP would be unique in that the various mechanisms have such widely different characteristics. Most propagation mechanisms, eg LOS, surface wave, troposcatter and ionoscatter are reasonably predictable and of a continuous nature; ionospheric skywave, sporadic $\mathrm{E}$ and ducting are less predictable and may be intermittent over a long timescale; meteor-burst propagation is intermittent over a very short timescale. Thus, the frequency management sub-system will make use of a mix of RTCE and embedded statistical modelling for link establishment and running control purposes. Some RTCE will take the form of active sounding in which sounding bandwidths will be varied, and probing carried out on

\subsection{4}


irregular schedules, via insertions within the normal traffic transmissions. Frequency change times will not exceed a few tens of ms.

\section{Concluding Remarks}

This paper has sought to describe the problems inherent in the design of radio communication systems to operate efficiently over MMPPs in the frequency range up to about $200 \mathrm{MHz}$. The problems are associated primarily with:

(i) path characterisation and modelling, including RTCE;

(ii) the nature and combination of adaptive channel encoding/decoding techniques appropriate to such paths;

(iii) the optimum use of digital processing and specialised DSP devices within the overall architecture of the radio system terminal.

Of particular interest is the potential usefulness of the lowfrequency troposcatter mode of propagation in the MMPP context.

Also indicated is the general nature of the solutions to the above problems.

A development of a 2-200 MHz MMPP system is being carried out in the UK by the Hull-Warwick Communications Research Group, sponsored by the Defence Research Agency (Farnborough). Transmitter powers of $200 \mathrm{~W}$ are being used.

\section{References}

[1] CCIR: "Ground-wave propagation curves for frequencies between $10 \mathrm{kHz}$ and $30 \mathrm{MHz}$ ", Rec. 368-3, ITU, Geneva, 1978.

[2] Davies, K.: "Ionospheric radio propagation", National Bureau of Standards (USA) Monograph No 80, April 1965.

[3] Whitehead, J.D.: "Survey of sporadic-E processes", Space Res. VII, North-Holland Publ. Co., Amsterdam, 1967.

[4] Oetting, J.D.: "An analysis of meteor-burst communication for military applications", IEEE Trans. Com munications, Vol. COM-28, No 9, September 1980.

[5] Bartholome, P.J., and Vogt, I.M.: "Ionoscatter communications: new design concepts and experimental results", SHAPE Technical Centre Report TR-53, April 1965 .
[6] Roda, G.: "Troposcatter radio links", Artech House, 1988 .

[7] Rotheram, S.: "Radiowave propagation in the evaporation duct", Marconi Review, Vol. 37, No 192, 1984.

[8] Forsse'n, K.G.: "Troposcatter data transmission tests at $30 \mathrm{MHz}$ ", Proc. Nordic Shortwave Conf. "HF-89", Faro, Sweden, August 1989.

[9] Darnell, M., Riley, N.G. and Melton, D.: "Beyond lineof-sight radio systems in the low-VHF band", Proc. 2nd Bangor Symp. on "Communications", Bangor, Wales, May 1990.

[10] Darnell, M. and Honary, B.: "Multi-functional coding schemes applicable to secure communication", Proc IEE Int Conf on "Secure communication systems", London, October 1986.

[11] Darnell, M and Honary, B.: "New synchronisation techniques applicable to multi-tone transmission systems", AGARD CP-442 on "Propagation effects and circuit performance of modern military radio systems with particular emphasis on those employing bandspreading", Paris, 1988.

[12] Darnell, M., Honary, B. and Zolghadr, F.: "Embedded coding technique: principles and theoretical studies", Proc. IEE-F, Vol. 135, No 1, 1988.

[13] Honary, B. and Darnell, M.: "Embedded modulation. and coding for HF channels", IEEE Int. Symp on "Information Theory", San Diego, USA, January 1990.

[14] Darnell, M.: "Embedded real-time channel evaluation techniques", AGARD LS-145 on "Propagation impact on modern HF communication system design", Belgium/France/Denmark, April 1986.

\section{Acknowledgement}

The authors gratefully acknowledge the support of DRA, Aerospace Division, (Farnborough) and SERC, (Science and Engineering Research Council), for the work described in this paper. 